\title{
IDDM2/insulin VNTR modifies risk conferred by IDDM1/HLA for development of Type 1 diabetes and associated autoimmunity
}

\author{
M. Walter ${ }^{1}$, E. Albert ${ }^{2}$, M. Conrad ${ }^{1}$, E. Keller ${ }^{2}$, M. Hummel ${ }^{1}$, K. Ferber ${ }^{1}$, B. J. Barratt ${ }^{3}$, J. A. Todd ${ }^{3}$, \\ A.-G. Ziegler ${ }^{1}$, E. Bonifacio ${ }^{4}$ \\ ${ }^{1}$ Diabetes Research Institute and 3rd Medical Department, Munich-Schwabing Hospital, Munich, Germany \\ ${ }^{2}$ Laboratory for Immunogenetics, Ludwig-Maximilians-University, Munich, Germany \\ 3 JDRF/WT Diabetes and Inflammation Laboratory, Cambridge Institute for Medical Research, University of Cambridge, \\ Cambridge, UK \\ ${ }^{4}$ Istituto Scientifico San Raffaele, Milan, Italy
}

\section{Abstract}

Aim/hypothesis. Type 1 diabetes (T1D) is an autoimmune disease with multiple susceptibility genes. The aim of this study was to determine whether combining IDDMI/HLA and IDDM2/insulin (INS) 5' variable number of tandem repeat locus (VNTR) genotypes improves T1D risk assessment.

Methods. Patients with T1D ( $n=488)$, control subjects $(n=846)$, and offspring of parents with T1D $(n=1122)$ were IDDMI and IDDM2 genotyped. Offspring were followed for islet autoantibodies and T1D from birth until the age of 2 to 12 years.

Results. Compared to the I/I INS VNTR genotype, the I/III and III/III genotypes reduced T1D risk conferred by IDDM1/HLA in all HLA genotype categories of the case-control cohort by 1.6 -fold to three-fold. The highest T1D risk was associated with INS VNTR class I/I plus HLA DR3/DR4-DQ8 (20.4\% in patients, 0.6\% in control subjects) or HLA DR4-DQ8/DR4-DQ8 (6.3\% in patients, $0.2 \%$ in control subjects). In the offspring, HLA DR3/DR4-DQ8 and DR4-DQ8/DR4DQ8 conferred increased risk for early development of islet autoantibodies $(14.6 \%$ and $12.9 \%$ by age 2 years). Offspring with these high risk IDDM1 genotypes plus the INS VNTR class I/I genotype $(n=71$; $6.3 \%$ ) had the highest risk of developing islet autoantibodies $(21.8 \%$ by age 2 years vs $8.9 \%$ in offspring with high risk IDDMI plus INS VNTR class I/III or III/III genotypes, $p<0.05)$ and T1D $(8.5 \%$ by age 6 years vs $4.3 \%$ ). Offspring who developed autoantibodies to multiple antigens had increased frequencies of both high risk IDDMI and IDDM2 genotypes $(p<0.0001)$, whereas offspring who developed autoantibodies to GAD only had increased frequencies of high risk $I D D M I$ and protective IDDM 2 genotypes, suggesting that IDDM2 influences the autoimmune target specificity.

Conclusion/Interpretation. Combining IDDMI and IDDM2 genotyping identifies a minority of children with an increased T1D risk. [Diabetologia (2003) 46:712-720]

Keywords IDDM1,IDDM2, HLA, insulin, autoantibodies.
Received: 2 October 2002 / Revised: 17 December 2002

Published online: 16 May 2003

(C) Springer-Verlag 2003

Corresponding author: Prof. A.-G. Ziegler, Diabetes Research Institute and 3rd Medical Department, Munich-Schwabing Hospital, Kölner Platz 1, 80804 Munich, Germany

E-mail: anziegler@1rz.uni-muenchen.de

Abbreviations: Abs, antibodies; dNTP, desoxynucleotide triphosphate; IAA, insulin autoantibodies; INS, insulin gene; OR, odds ratio; T1D, Type 1 diabetes; VNTR, variable number of tandem repeat.
Type 1 diabetes (T1D) is an autoimmune disease with multiple susceptibility genes $[1,2]$. The major susceptibility genes are found within the HLA class II region of chromosome 6 (IDDMI). A second genetic susceptibility locus has been mapped by a variable number of tandem repeat (VNTR) in the insulin gene (INS) promoter region $(I D D M 2)[3,4]$. Identification of T1D-prone individuals through genotyping would facilitate prospective investigation of early environmental triggers and primary intervention trials. Such studies would become feasible if they could include only subjects with highest T1D risk and if early outcome 
markers were identified. Several prospective studies have shown that the islet autoimmunity that precedes T1D onset can be seen very early in life and can be used as an outcome marker to study triggers of autoimmunity [5, 6, 7]. We, and others, have shown that IDDM1 genotyping can identify newborns with an increased risk of developing T1D-associated autoimmunity $[8,9,10]$. Here we determine whether combining IDDM 1 and IDDM 2 genotyping improves risk assessment. We identify IDDMI and IDDM2 T1D risk genotypes in a case-control cohort from Germany, and subsequently evaluate the predictive value of genetic pre-screening by follow-up for autoimmunity and T1D within the prospective BABYDIAB newborn offspring study. By combining IDDMI and IDDM2 genotyping it was possible to identify a minority of newborns of parents with T1D who had a greater than $20 \%$ risk to develop persistent multiple islet autoantibodies by the age of 2 years and almost $10 \%$ risk to develop T1D by the age of 6 years. These findings allow primary intervention programs to delay T1D onset to be seriously considered and designed.

\section{Subjects and Methods}

Subjects. DNA samples were obtained from a total of 538 Caucasian patients with T1D from Germany. These were parents of children who participated in the BABYDIAB study [5]. Their mean (SD) age of T1D onset was $17.5(+8.5)$ years, 397 were female, 66 had a first degree relative (parent or sibling) with T1D, and $53 \%$ were living in the northern half of Germany (Zip code 01558-59889). The female bias in patients was due to a recruitment restriction in the first few years of the BABYDIAB study allowing only families in which mothers had T1D to participate. As control subjects, DNA was obtained from the non-diabetic parents of the BABYDIAB children $(n=610)$ and from 307 unrelated non-diabetic volunteers without family history of T1D (45\% were females and 55\% were living in the northern half of Germany). DNA samples were also obtained from 1249 offspring of mothers $(n=785)$, fathers $(n=436)$, or both parents $(n=28)$ with T1D. All offspring were participating in the German BABYDIAB study and were prospectively followed from birth in regular intervals up to the age of 12 years (median follow-up time 5.6 years; range 1.3-12.3 years) [5]. During follow-up T1D-associated islet autoantibodies [insulin autoantibodies (IAA), GAD autoantibodies, antibodies to the protein tyrosine phosphatase IA-2, and islet cell autoantibodies] were measured at 9 months, 2 years, 5 years, 8 years, and 11 years of age. Children with positive autoantibodies were followed with blood samples at 6 to 12 month intervals. Of the 1249 offspring, 63 have developed persistent (positive in at least two consecutive samples) islet autoantibodies, including 40 with autoantibodies to more than one antigen (multiple autoantibodies). Of these 63, 19 developed persistent islet autoantibodies by 9 months, an additional 25 by 2 years, an additional 15 by 5 years, and an additional 4 by 8 years. 18 offspring with persistent islet autoantibodies and none of the islet autoantibody negative offspring have developed T1D. IDDM1 HLA DR-DQ genotyping and IDDM2 INS VNTR genotyping was carried out on 488 T1D patients, 846 control subjects and 1122 offspring. The remaining 50 patients, 71 control subjects and 127 offspring were
IDDM1 HLA DR-DQ genotyped only. Written informed consent was obtained from all subjects who participated in the study. The study was approved by the Bayerische Landesärztekammer ethical committee (Nr. 95357).

HLA typing. HLA-DRB1-DQA1 and -DQB1 alleles were typed using polymerase chain reaction (PCR)-amplified DNA and non-radioactive oligonucleotide probes (sequence-specific oligonucleotide typing SSO) in the laboratories of Ekkehard Albert (Ludwig-Maximilians-University, Munich). Genomic DNA was isolated from fresh peripheral blood cells (EDTAblood) and diluted to $250 \mu \mathrm{g} / \mathrm{ml}$ in TE buffer and stored at $-20^{\circ} \mathrm{C}$ until use. Genomic DNA $(1 \mu \mathrm{g})$ was amplified by PCR using DRB1, DQB1 or DQA1 generic primers, spotted onto nylon membranes (Roche, Mannheim, Germany) and hybridized with 15 DRB1-, 17 DQA1- or 20 DQB1-allele-specific oligonucleotides, respectively. The hybridized oligonucleotides 3 'end labelled with digoxigenin-11-2',3'-dideoxyuridine-5'-triphosphate (Roche) were detected using anti-dioxigenin-AP Fab fragments (Roche) and visualized with the chemiluminescent substrate CSPD (Roche).

INS VNTR. Subjects were typed using HphI digestion of PCR amplification products of the region of interest as previously described [3]. PCR amplification was carried out in 96-well microtitre plates. Each $25 \mu \mathrm{l}$ reaction tube contained $100 \mathrm{ng}$ DNA, $2.5 \mu \mathrm{l} 10 \times$ reaction buffer (Eppendorf, Hamburg, Germany), $200 \mathrm{mmol} / \mathrm{l}$ desoxynucleotide triphosphate (dNTP)Mix, 1.0 ng from each primer, 5.0 $\mu \mathrm{l}$ Taq-Enhancer (Eppendorf) and $0.5 \mathrm{U}$ of Taq-Polymerase (Eppendorf). The PCR was carried out in 32 cycles $\left(94^{\circ} \mathrm{C} 20 \mathrm{~s}, 57^{\circ} \mathrm{C} / 15 \mathrm{~s}, 72^{\circ} \mathrm{C} / 30 \mathrm{~s}\right)$. Primers for PCR were: $23 \mathrm{HphI} 360 \mathrm{~F} 5^{\prime}$-AGCAGGTCTGTTCCAAGG-3' and $23 \mathrm{HphI}$ 360R 5'-CTTGGGTGTGTAGAAGAAGC-3'. $25 \mu \mathrm{l}$ of each PCR product was digested in $2 \mathrm{U}$ of $H p h \mathrm{I}$ at $37^{\circ} \mathrm{C}$ for $2 \mathrm{~h}$, and each digested product was detected by ethidium bromide staining after electrophoresis in a $3 \%$ NuSieve gel (FMC BioProducts, Rockland, Me., USA).

Islet Autoantibody. Testing Autoantibodies to insulin (IAA), GAD, and IA-2 were measured in offspring samples by radiobinding assays [5, 7]. The upper limit of normal was defined by q-q plots [5]. Using these thresholds for positivity, the assays had sensitivities and specificities of $80 \%$ and $94 \%$ (GAD antibodies), $58 \%$ and $100 \%$ (IA-2 antibodies), and $30 \%$ and 98\% (IAA) in the First DASP Proficiency [11].

Statistical analysis. Allele, haplotype, and genotype frequencies were calculated for patients and the control group. Where both parents and offspring were typed, haplotypes were unambiguously defined by transmission. In families where unambiguous haplotype could not be defined, haplotypes were assigned by known HLA-DRB1-DQA1-DQB1 combinations. Odds Ratios and their $p$ values were calculated by logistic regression, using Stata Statistical Software, Release 7.0 (Stata, College Station, Tex., USA). The Chi-square test for trend was used to compare genotype frequencies between age groups. Fisher's exact test was used to compare genotype frequencies between multiplex and single case families. Life table analysis was used to calculate the cumulative islet autoantibody frequency (positive predictive value) [8] using the Statistical Package for Social Science (SPSS, Chicago, Ill., USA). A $p$ value of less than 0.05 was considered statistically significant. 


\section{Results}

HLA-DRB1-DQA1-DQB1 genotypes in patients, controls and offspring cohorts. HLA-DRB1-DQA1DQB1 genotypes conferring T1D susceptibility or protection in the case-control cohort are shown in Table 1. Patients who developed T1D before the age of 10 years $(n=104)$ had a higher frequency of the very high risk DRB1*03-DQA $1 * 0501-\mathrm{DQB} 1 * 0201 /$ DRB1*04-DQA1*0301-DQB1*0302 (DR3/DR4-DQ8) genotype than patients who developed T1D after the age of 10 years $(36.5 \%$ vs $24.1 \%, p<0.02$, Table 2$)$. Patients who had first degree relatives with T1D $(n=66)$ had a higher frequency of the high risk DRB 1*04-DQA $1 * 0301-D Q B 1 * 0302 / D R B 1 * 04-D Q A 1$ *0301-DQB1*0302 (DR4-DQ8/DR4-DQ8) genotype than patients without a family history of T1D (18.1\% vs $8.1 \%, p<0.05)$. No differences were observed in genotype frequencies between male and female patients (very high risk: $22.7 \%$ vs $28.0 \%$; high risk: 9.2\% vs $9.3 \%$; moderate DR4-DQ8: $15.6 \%$ vs $15.6 \%$; Moderate DR3:13.5\% vs $9.8 \%$; neutral: $29.8 \%$ vs $30.7 \%$; protective: 9.2 vs $6.5 \%$ ) or between male and female control subjects. Within the case-control cohort, DQ8 conferred a lower susceptibility when to- gether with DRB1*0402, DRB1*0403, or DRB 1*0404 alleles than with DRB1*0401 (all $p<0.02$; data not shown).

The high-risk HLA DR3/DR4-DQ8 and DR4DQ8/DR4-DQ8 genotypes were found in 155 (8\% and $4.4 \%$, respectively) of 1249 offspring from patients with T1D. A further 122 (9.8\%) offspring had DR4DQ8 genotypes conferring moderate T1D risk, and 61 (4.9\%) offspring had DR3 genotypes conferring moderate risk (Table 1). DQ8 haplotypes with the less susceptible DRB $1 * 04$ alleles were found in $20(* 0402)$, $4(* 0403)$, and $41(* 0404)$ of the 1249 offspring (data not shown).

INS VNTR genotype frequency. The INS VNTR class I allele frequency in patients was $86.5 \%$ compared with $75 \%$ in control subjects $(p<0.0001)$. The I/I, I/III, and III/III genotypes were found in $75 \%, 23.2 \%$, and $1.8 \%$ of patients with T1D compared to $56 \%, 37.2 \%$, and $6.8 \%$ of control subjects $(p<0.0001$; Table 2$)$. This difference was similar in males $(78.1 \%, 19.5 \%, 2.3 \%$ in patients vs $54.9 \%, 38.3 \%, 6.8 \%$ in control subjects, $p<0.0001)$ and females $(73.9 \%, 24.1 \%, 1.7 \%$ in patients vs $57.5 \%, 35.2 \%, 7.3 \%$ in control subjects, $p<0.0001)$. T1D risk was reduced for both class I/III

Table 1. HLA DR-DQ genotype frequency in patients with T1D and in BABYDIAB offspring from patients with T1D

\begin{tabular}{|c|c|c|c|c|c|c|c|}
\hline & 03/04 & 02/0302 & 26.6 & 1.2 & 21.1 & $<10^{-19}$ & 8.0 \\
\hline \multicolumn{8}{|l|}{ High risk } \\
\hline \multicolumn{8}{|c|}{ Moderate DR4-DQ8 } \\
\hline & 04/08 & 0302/0402 & 2.4 & 0.3 & 7.1 & $<10^{-2}$ & 1.6 \\
\hline & $04 / 13$ & 0302/0604 & 4.1 & 0.8 & 5.1 & $<10^{-3}$ & 1.5 \\
\hline & $04 / 16$ & $0302 / 0502$ & 1.1 & 0.2 & 4.9 & 0.05 & 1.3 \\
\hline & $04 / 01$ & 0302/0501 & 8.0 & 2.4 & 3.2 & $<10^{-4}$ & 5.4 \\
\hline \multicolumn{8}{|l|}{ Neutral } \\
\hline & rest & & 30.5 & 29.0 & $1.0 *$ & & 39.3 \\
\hline \multicolumn{8}{|l|}{ Protective \# } \\
\hline & $\mathrm{Z} / 11$ or 12 & z/0301 & 2.5 & 23.5 & 0.16 & $<10^{-8}$ & 10.1 \\
\hline & $\mathrm{Z} / 07$ & z/0303 & 0.6 & 6.8 & 0.11 & $<10^{-3}$ & 2.6 \\
\hline & $\mathrm{Z} / 13$ & z/0603 & 1.1 & 12.8 & 0.10 & $<10^{-7}$ & 6.6 \\
\hline & $\mathrm{Y} / 14$ & $\mathrm{x} / 0503$ & 0.4 & 5.5 & 0.08 & $<10^{-3}$ & 3.0 \\
\hline & $\mathrm{Y} / \mathrm{Y}$ & $\mathrm{x} / 0602$ & 2.6 & 26.4 & 0.09 & $<10^{-15}$ & 13.0 \\
\hline
\end{tabular}

*reference genotype; $\mathrm{x}=$ any DQB1 allele; $\mathrm{Y}=$ any DRB1 allele; Z-Z = any DRB1-DQB1 haplotype except DRB1*04DQB1*0302. \#Subjects who have two protective haplotypes (eg DR11-DQB1*0301 and DR7-DQB1*0303) are represented in each of the protective genotypes 
Table 2. IDDMIHLA risk genotype and IDDM2 INSVNTR genotype in patients with T1D, control subjects and BABYDIAB offspring

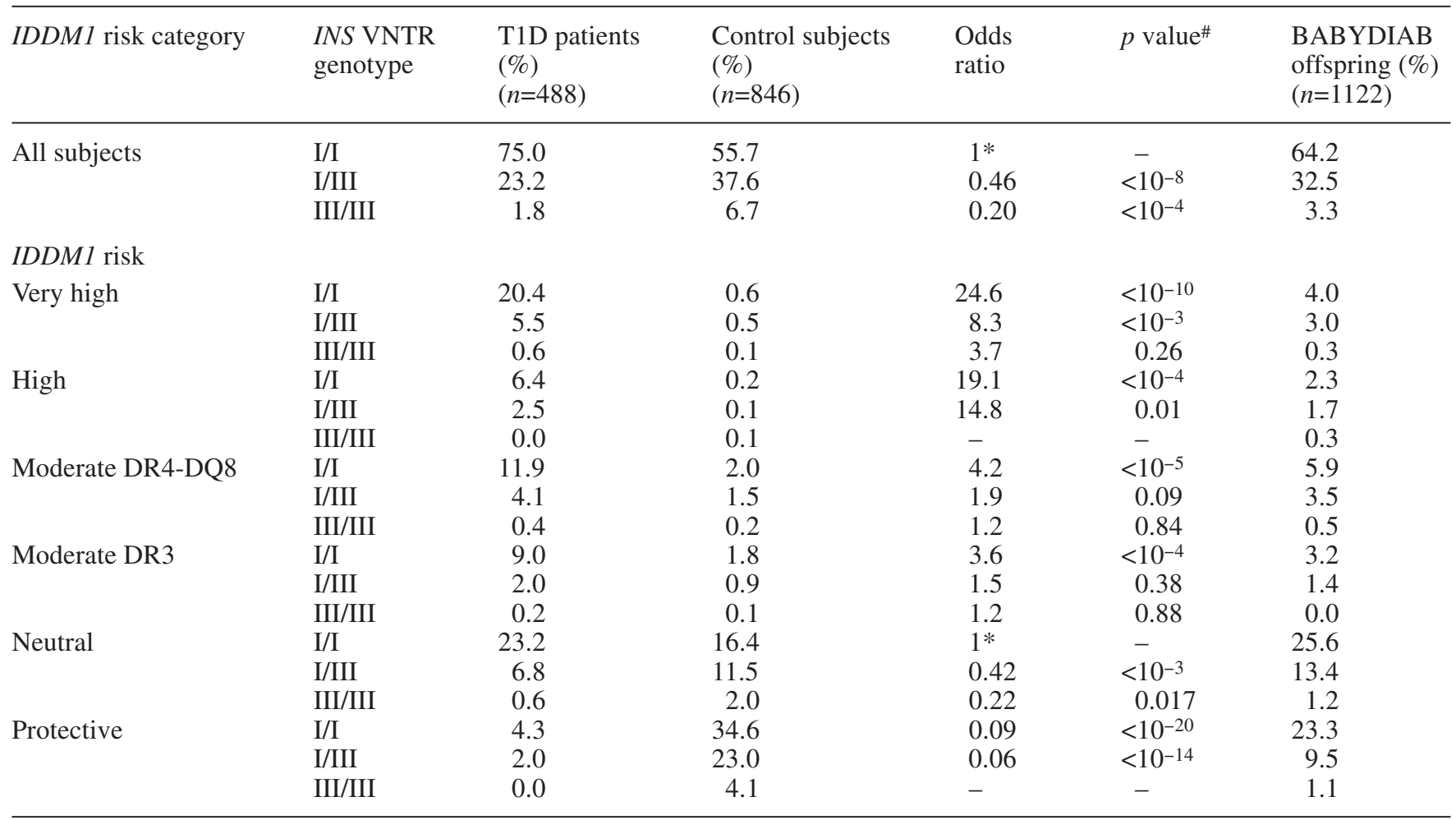

* reference genotype, ${ }^{\#} p$ values are uncorrected

Table 3. Cumulative frequency of persistent islet autoantibodies and T1D in BABYDIAB offspring: IDDM1genotype

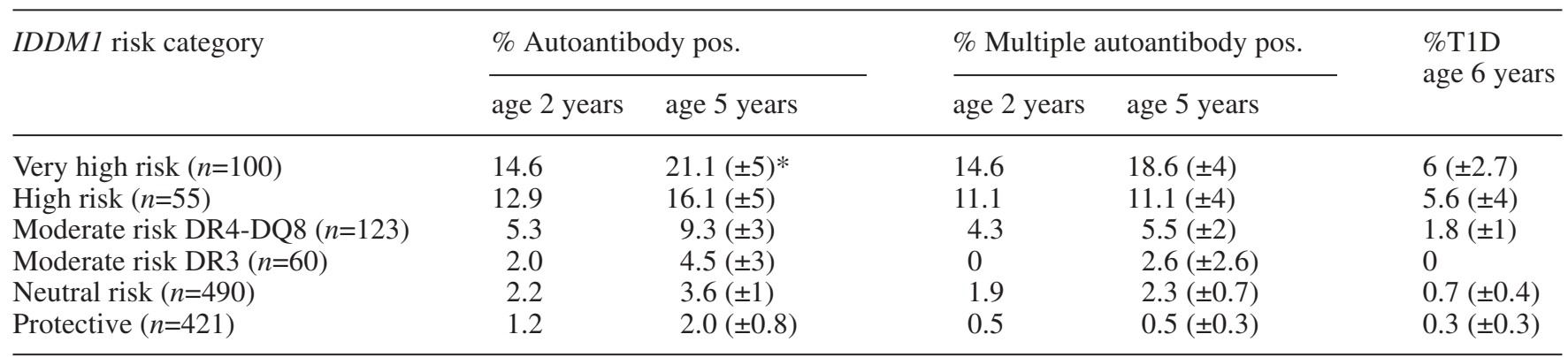

*standard error

(OR 0.46) and III/III (OR 0.2) in comparison to class I/I genotypes suggesting that the class III allele confers semi-dominant protection. INS VNTR genotype frequencies in patients did not differ with respect to age of diabetes onset, or family history of T1D (data not shown).

The INS VNTR genotype modified T1D risk in all HLA DR-DQ genotype risk categories (Table 2). The INS VNTR class I/III and III/III genotypes were associated with a decreased T1D risk compared to INS VNTR I/I in the presence of HLA DR-DQ genotypes conferring very high and high T1D risk (OR 0.32; $p<0.05$ class I/III or III/III vs class I/I), moderate T1D risk (OR 0.43; $p=0.01$ ), neutral T1D risk (OR 0.39; $p<0.0001$ ), or protection (OR $0.61 ; p=0.21$ ). Modifi- cation of T1D risk by INS VNTR in the different HLA DR-DQ risk categories was observed in both males $(\mathrm{OR}=0.24,0.26,0.41$, and 0.61 for INS VNTR class I/I vs I/III or III/III in HLA high risk, moderate risk, neutral, or protective genotypes, respectively) and females (ORs of $0.56,0.50,0.32$, and 0.62 for INS VNTR class I/I vs I/III or III/III in HLA high risk, moderate risk, neutral, or protective genotypes, respectively).

HLA-DRB1-DQA1-DQB1-INS VNTR genotypes and autoantibody/T1D risk in offspring. The cumulative frequency of islet autoantibodies and T1D in offspring according to HLA-DRB1-DQA1-DQB1 genotype is shown in Table 3 and Fig. 1. Autoantibody frequency was highest in offspring with the HLA DR3/DR4-DQ8 
A. Islet autoAbs

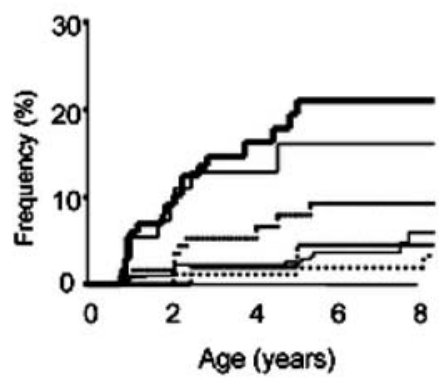

B. Multiple islet autoAbs

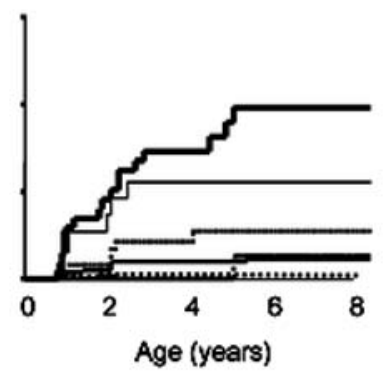

C. T1D

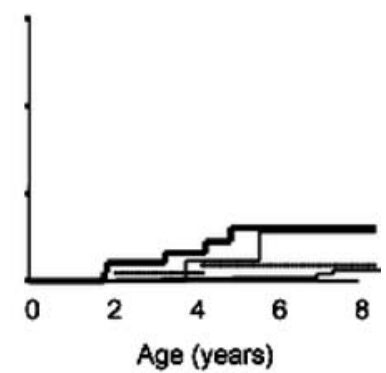

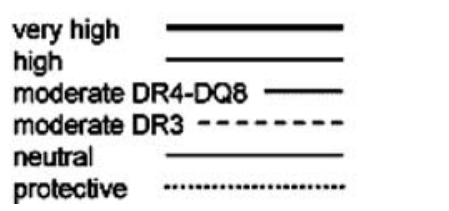

shown by the dotted line. Offspring with very high, high, and moderate DR4 risk genotypes had increased risk for developing islet autoantibodies $(p<0.0001, p<0.0001, p<0.005$ vs remainder) and multiple islets autoantibodies $(p<0.001, p<0.0001$, $p<0.01$ vs remainder). T1D risk was increased in offspring with very high $(p<0.0001)$ and high $(p<0.001)$ risk genotypes

A. Islet autoAbs
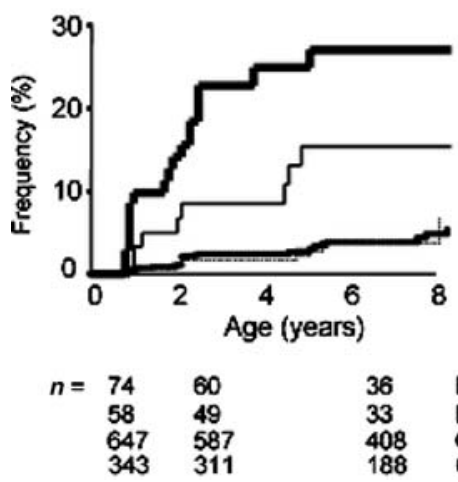

Fig. 2A-C. Life table analysis of developing at least one persistent islet autoantibody (A), at least two persistent islet autoantibodies (B), or Type 1 diabetes (C) in offspring of parents with Type 1 diabetes in the BABYDIAB study. Offspring are grouped by HLA-DR-DQ and INS VNTR genotype. Islet autoantibody frequencies are higher in offspring with high risk HLA DR-DQ and INS VNTR class I/I genotypes than offspring with high risk HLA DR-DQ and INS VNTR class I/III or III/III genotypes $(p<0.05)$ or offspring with other genotypes $(p<0.0001)$

(21.1\% by age 5 years) and DR4-DQ8/DR4-DQ8 ( $16.1 \%$ by age 5 years) genotypes $(p<0.0001$ vs $3.9 \%$ in offspring with other genotypes). Antibody frequency was also increased in offspring with the DR4-DQ8 containing moderate risk genotypes $(9.3 \%$ by age 5 years; $p<0.005$ vs $3.1 \%$ in offspring with neutral and protective), but not in offspring with the moderate DR3 risk genotypes. Autoantibody frequency was highest in DR3/DR4-DQ8 or DR4-DQ8/DR4-DQ8 offspring who had the INS VNTR class I/I genotype (Table 4, Fig. 2). A total of $71(6.3 \%)$ of 1122 offspring had these com- bined DRB1-DQA1-DQB1-INS VNTR genotypes. These 71 offspring had a $23.1 \%$ cumulative frequency of persistent autoantibodies already by the age of 2 years, a $21.1 \%$ frequency of persistent multiple antibodies by the age of 2 years, and an $8.5 \%$ cumulative frequency of T1D by the age of 6 years. Autoantibody frequency was higher in offspring with high risk DRB1-DQA1-DQB1-INS VNTR genotypes than those with only the high risk DRB1-DQA1-DQB1 genotypes $(p<0.05)$. This difference was similar in children of mothers with T1D (21.1\% cumulative frequency of autoantibodies by age 2 years in children with high risk DRB1-DQA1-DQB1-INS VNTR genotypes vs $8.4 \%$ in children with only the high risk DRB1-DQA1-DQB1 genotypes) and children of fathers with T1D (25.1\% vs 8.5\%; data not shown). INS-VNTR genotypes did not modify risk of developing islet autoantibodies in the offspring with moderate, neutral or protective IDDMI HLA genotypes $(2.6 \%$ by age 2 years in offspring with INS VNTR I/I vs $2.1 \%$ in offspring with I/III of III/III), but numbers of autoantibody positive cases in these offspring were low. 


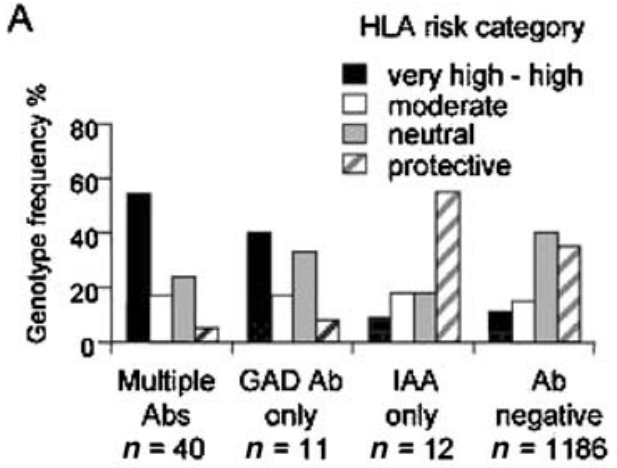

B

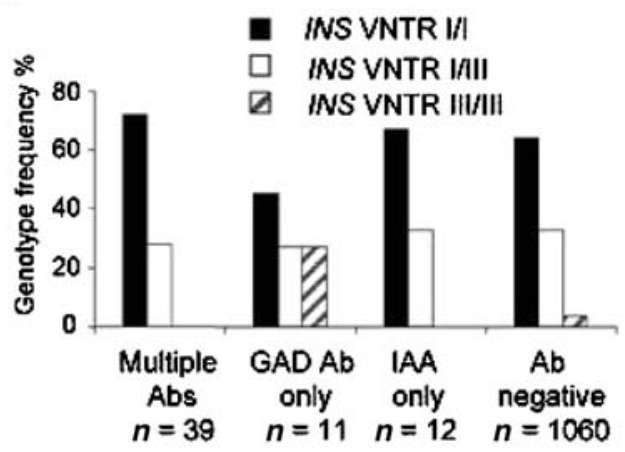

C

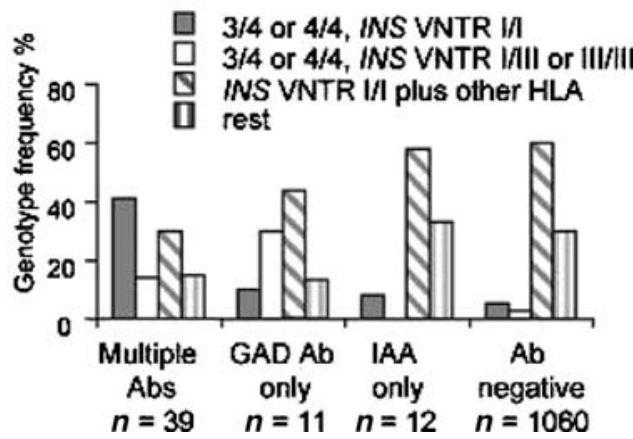

Fig. 3A-C. Frequency of IDDMI HLA DR-DQ genotypes (A), and IDDM2 INS VNTR genotypes (B) and combined IDDMI HLA DR-DQ and IDDM2 INS VNTR T1D risk genotypes $(\mathbf{C})$ in offspring with multiple persistent islet antibodies, in offspring with persistent GADA only, in offspring with persistent IAA only, and in islet antibody negative offspring. Offspring with persistent multiple autoantibodies $(p<0.0001$ vs antibody negative) or GAD autoantibodies only $(p<0.02)$ had increased prevalences of the DR3/DR4-DQ8 and DR4-DQ8/DR4-DQ8 genotypes (A). The INS VNTR class III/III was more prevalent in offspring with GAD antibodies only ( $p<0.02$ vs IAA positive, $p<0.01$ vs antibody negative) (B). Multiple autoantibody positive offspring had an increased prevalence of DR3/DR4-DQ8 or DR4DQ8/DR4-DQ8 plus INS VNTR I/I genotypes $(p<0.0001$ vs autoantibody negative offspring) (C); Offspring with GAD antibodies only had an increased prevalence of DR3/DR4-DQ8 or DR4-DQ8/DR4-DQ8 plus INS VNTR I/III or III/III genotypes $(p<0.005$ vs antibody negative offspring) $(\mathbf{C})$

HLA-DRB1-DQA1-DQB1-INS VNTR genotypes and autoantibody target antigen. Persistent non-maternally acquired islet autoantibodies developed in 63 of the HLA typed offspring: 18 of these had the HLA
DR3/DR4-DQ8, and eight the HLA DR4-DQ8/DR4DQ8 genotypes, nine had protective and 16 neutral genotypes. Offspring who developed persistent autoantibodies to more than one antigen $(n=40)$ had similar DRB1-DQA1-DQB1 genotypes to patients with T1D onset with an increase in DR3/DR4-DQ8 and DR4-DQ8/DR4-DQ8 genotypes $(p<0.0001$ vs antibody negative offspring; Fig. 3A). Children who developed GAD antibodies only $(n=11)$ also had a higher prevalence of high risk HLA genotypes $(p<0.02)$. In contrast, offspring who developed persistent IAA only $(n=12)$ had remarkably similar $H L A$ $D R B 1-D Q A 1-D Q B 1$ genotypes to offspring who remained autoantibody negative and control subjects. Most (7 of 9) islet autoantibody positive offspring with protective $H L A-D R B 1-D Q A 1-D Q B 1$ genotypes had IAA only. IAA in these offspring were not maternally acquired (developed at or after age 2 years), were persistent, and were similar in titre to IAA in offspring with multiple islet autoantibodies (data not shown). INS VNTR genotypes also showed differences between antibody positive offspring (Fig. 3B). Notably, the protective INS VNTR class III/III genotype was found in $3(27 \%)$ of 11 offspring with GAD antibodies only and in none of 51 offspring with IAA $(p<0.02$ GAD antibody only vs $0 \%$ in multiple or IAA only; $p<0.01$ vs $3.3 \%$ in autoantibody negative offspring).

The number and target specificity of autoantibodies was influenced by the combination of IDDMI and IDDM2 genotypes (Fig. 3C). Multiple autoantibodies were associated with both IDDMI and IDDM2 high risk genotypes: 16 of 39 offspring with multiple autoantibodies (all IAA and GAD antibody positive) had both IDDMI (HLA DR3/DR4-DQ8 or DR4-DQ8/ DR4-DQ8) and IDDM2 (INS VNTR class I/I) highrisk genotypes $(p<0.0001$ versus autoantibody negative offspring). Offspring who developed autoantibodies to GAD only had an increased prevalence of highrisk IDDMI genotypes in the absence of high-risk IDDM 2 genotypes (27\% versus $2.7 \%$ in autoantibody negative offspring; $p<0.005)$. IDDMI and IDDM2 genotypes in offspring with persistent IAA only were similar to those in the autoantibody negative offspring and showed no enrichment of the high-risk genotypes. IA-2 antibodies were only found in offspring with multiple antibodies and were associated with both high risk $I D D M 1$ and $I D D M 2$ genotypes.

\section{Discussion}

This study classifies T1D risk on the basis of IDDMI and IDDM2 genotyping. It identifies and stratifies individual HLA class II genotypes conferring different risks for T1D, confirms the separate contribution of the HLA-DRB1 locus to T1D risk, and demonstrates that the IDDM2 gene confers additional risk above 
Table 4. Cumulative frequency of persistent islet autoantibodies and T1D in BABYDIAB offspring: IDDMIand IDDM2 genotype

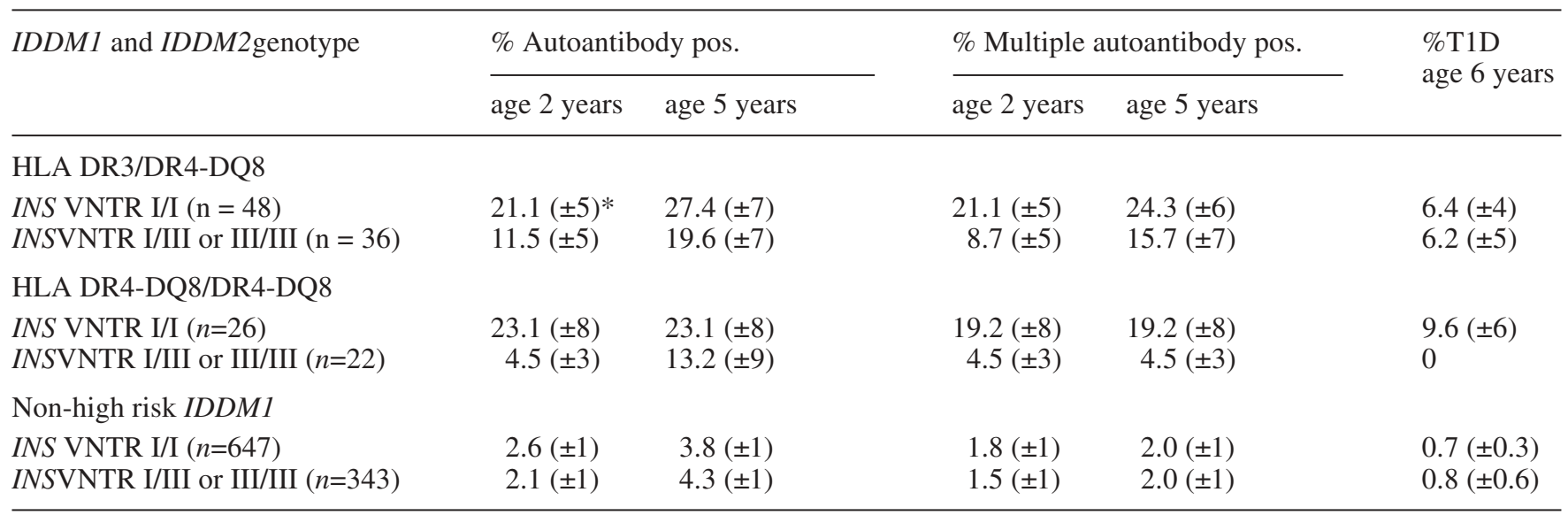

*standard error

that of IDDMI, regardless of IDDMI genotype. Follow-up of over 1200 offspring of parents with T1D demonstrated that early T1D-associated autoimmunity and T1D was frequent only in children who had the high-risk IDDMI genotypes and was most frequent in children who had combinations of both high risk IDDM 1 and IDDM 2 genotypes.

High risk $I D D M 1$ genotypes in Germany were similar to those of other Caucasian populations $[12,13$, $14,15,16]$. Consistent with the high T1D risk conferred by the DR3/DR4-DQ8 and DR4-DQ8/DR4DQ8 genotypes, a markedly increased risk of developing islet autoantibodies in the first years of life was observed for DR3/DR4-DQ8 or DR4-DQ8/DR4-DQ8 offspring of parents with T1D. The marked risk in the offspring with HLA-DR4-DQ8/DR4-DQ8 (similar to that of DR3/4) is consistent with the high prevalence of this genotype found in T1D patients who had a first degree relative with T1D. A novel finding was that offspring with other DR4 containing T1D risk genotypes, but not those with DR3 containing risk genotypes had an increased likelihood of developing islet autoantibodies.

IDDM2 genotyping was done in order to determine whether its addition to IDDMI genotyping might improve prediction of offspring who will develop T1D. The contribution of IDDM2 to T1D risk has been consistently demonstrated by others $[3,17,18,19]$. However, it remains unclear whether risk is modified equally in subjects with different IDDMI genotypes. Data from the Belgium diabetes registry showed that IDDM2 conferred increased risk only in subjects with moderate risk HLA DR-DQ genotypes suggesting that there was interaction between these genes and that INS VNTR typing in subjects with high-risk IDDMI genotypes would be of limited value [20]. Our data on a larger patient and control cohort found modification of risk in subjects with the high risk and neutral HLADR-DQ genotypes. Risk modification was similar in all HLA DR-DQ genotypes, but numbers of patients with protective HLA genotypes and of controls with the high risk genotypes were too small to allow meaningful comparisons. We therefore cannot exclude that IDDM 2 modifies risk less in subjects with very high risk IDDM1 genotypes than in subjects with moderate risk genotypes, as suggested by the Belgian data. Within subjects with the high risk IDDMI HLA DR3/DR4-DQ8 genotype, the INS VNTR class I/III or III/III genotypes was associated with more than twofold lower T1D risk than the class I/I genotype. Moreover, risk for T1D-associated autoantibody development in offspring was more than two-fold lower in DR3/DR4-DQ8 or DR4-DQ8/DR4-DQ8 offspring who also carried the INS VNTR class I/III or III/III genotypes compared to those who had the class I/I genotypes. Autoantibody risk in the offspring without high risk HLA genotypes was not modified by the INS VNTR genotypes. However, numbers of autoantibody offspring with non-high risk HLA risk genotypes was low and it remains to be determined whether INS VNTR can stratify risk in these offspring.

The class III allele of IDDM2 has been suggested to lower T1D risk by increasing immune tolerance to insulin and its precursors via increasing expression of the autoantigen in the thymus [21, 22]. The suggested model implies that insulin autoimmunity and therefore IAA would develop more frequently in individuals with the INS VNTR class I/I genotype than in individuals with the class I/III or III/III genotypes. Evidence in support of this was recently provided by the finding that the number of INS VNTR class I alleles was directly proportional to the prevalence of IAA at T1D onset [23]. The BABYDIAB offspring cohort provided a unique opportunity to test the hypothesis since autoantibodies were measured prospectively throughout the pre-diabetic period. This allowed assessment of genetic associations with the development of IAA per se and not just in those who subsequently develop T1D. IAA are the earliest autoantibodies that are detected in this cohort of children [5]. These are not ma- 
ternally acquired antibodies and are persistent in the majority of cases [7]. Most children who develop IAA often also develop antibodies to GAD and/or IA-2 (multiple islet antibodies), and these children have the highest T1D risk. A minority of children develop GAD antibodies without ever becoming IAA positive. We therefore compared IDDMI and IDDM2 genotypes in children with multiple islet autoantibodies (IAA and GAD antibody positive) to those with single antibodies and to islet autoantibody negative children. Children with both IAA and GAD antibodies had similar HLA and INS VNTR genotypes to patients with T1D. Multiple islet antibodies developed most frequently when offspring had both high risk IDDMI and INS VNTR class I/I genotypes. Children with GAD antibodies but no IAA also had an increase in high risk IDDMI genotype frequency, but in contrast to IAA positive children, had an excess of the protective INS VNTR class III/III genotype. This genotype was in $27 \%$ (3/11) of children with GAD antibodies only, but was not found in any of the 51 IAA positive children. Although the number of subjects developing GAD antibodies only was small, these data suggest that the development of IAA is prevented in the absence of INS VNTR class I alleles and are consistent with the hypothesis proposed by the other studies. The new finding that one copy of the class III INS VNTR reduces risk of disease and autoantibody development, with two copies of this allele providing a further risk reduction is also consistent with a peptide-HLA class II-T cell receptor affinity mechanism underlying the association of IDDMI/HLA with T1D and its relationship with IDDM2/INS VNTR [14, 21, 22]. Previously, it was concluded that IDDM2 was a recessive susceptibility locus or a dominant protective locus [3], but this is clearly not the case, as shown in a large family study (I.A. Eaves, B.J. Barratt, J.A. Todd, in preparation). An additional surprising finding was that the development of IAA without spreading to GAD or IA-2 antibodies was not associated with T1D risk IDDMI genotypes. This implies that persistent IAA in isolation are unlikely to be associated with high T1D risk.

The modification of T1D risk conferred by IDDM2 to IDDMI for developing T1D-associated autoimmunity to multiple autoantigens should improve our ability to identify at risk subjects from an early age. Several studies are under way to intensively follow, or to undertake primary prevention trials in, children at high risk for T1D $[5,6,9,10,24]$. Selection of children to be included into these studies is likely to be benefited by increased specificity since both intensive follow-up and primary intervention can be a burden to the family. The risk of T1D even for DR3/DR4-DQ8positive children can be substantially reduced by possession of one or two INS VNTR class III alleles, and it is anticipated that protective alleles at other disease loci will soon be identified, such as CTLA4 (J.A. Todd, unpublished). It is, therefore, conceivable that a combination of protective alleles of non-MHC loci in certain individuals could reduce the T1D risk of the HLA-DR3/DR4-DQ8 genotype to 1, thereby providing a case for their exclusion from a clinical trial. By combining family history of T1D, IDDMI and IDDM2 genotyping we were able to identify a cohort of children who had an $8.7 \%$ likelihood of developing T1D already by the age of 6 years and $21 \%$ risk for developing persistent islet autoimmunity to multiple T1D autoantigens already by the age of 2 years. This represents a high risk group that might be targeted in primary intervention trials when suitable candidate intervention therapies become available.

Acknowledgements. This study was supported by grants from the Juvenile Diabetes Research Foundation (JDRF \#1-2000619, 061858), the Wellcome Trust (061858), the Stiftung 'Das Zuckerkranke Kind', and the Deutsche Diabetesgesellschaft (Dr. Buding-Stiftung). We thank A. Knopff, J. Vordemann, I. Zöbisch and E. Kleinschmidt for data collection and expert technical assistance, and $\mathrm{H}$. Cordell for advice in statistical analyses. We also thank the pediatricians and family doctors in Germany for participation in the BABYDIAB study.

\section{References}

1. Cox NJ, Wapelhorst B, Morrison VA et al. (2001) Seven regions of the genome show evidence of linkage to Type 1 diabetes in a consensus analysis of 767 multiplex families. Am J Hum Genet 69:820-830

2. Redondo MJ, Eisenbarth GS (2002) Genetic control of autoimmunity in Type I diabetes and associated disorders. Diabetologia 45:605-622

3. Bennett ST, Lucassen AM, Gough SC et al. (1995) Susceptibility to human type 1 diabetes atIDDM2 is determined by tandem repeat variation at the insulin gene minisatellite locus. Nat Genet 9:284-292

4. Cordell HJ, Todd JA, Bennett ST, Kawaguchi Y, Farrall M (1995) Two-locus maximum lod score analysis of a multifactorial trait: joint consideration ofIDDM2 and IDDM4 with IDDM1 in type 1 diabetes. Am J Hum Genet 57:920-934

5. Ziegler AG, Hummel M, Schenker M, Bonifacio E (1999) Autoantibody appearance and risk for development of childhood diabetes in offspring of parents with type 1 diabetes: the 2-year analysis of the German BABYDIAB Study. Diabetes 48:460-468

6. Yu L, Robles DT, Abiru N et al. (2000) Early expression of antiinsulin autoantibodies of humans and the NOD mouse: evidence for early determination of subsequent diabetes. Proc Natl Acad Sci USA 97:1701-1706

7. Naserke HE, Bonifacio E, Ziegler AG (2001) Prevalence, characteristics and diabetes risk associated with transient maternally acquired islet antibodies and persistent islet antibodies in offspring of parents with type 1 diabetes. J Clin Endocrinol Metab86:4826-4833

8. Schenker M, Hummel M, Ferber K et al. (1999) Early expression and high prevalence of islet autoantibodies for DR3/4 heterozygous and DR4/4 homozygous offspring of parents with Type I diabetes: The German BABYDIAB study. Diabetologia 42:671-677

9. Yu J, Yu L, Bugawan TL et al. (2000) Transient antiislet autoantibodies: infrequent occurence and lack of association with "genetic" risk factors. J Clin Endocrinol Metab $85: 2421-2428$ 
10. Kupila A, Keskinen P, Simell T et al. (2002) Genetic risk determines the emergence of diabetes-associated autoantibodies in young children. Diabetes 51:646-651

11. Bingley PJ, Bonifacio E, Mueller P and participating laboratories (2001) Diabetes antibody standardisation programme: first assay proficiency evaluation Diabetologia 44 [Suppl. 1]:A 74 (Abstract)

12. Zavattari P, Lampis R, Motzo C et al. (2001) Conditional linkage disequilibrium analysis of a complex disease superlocus, IDDMI in the HLA region, reveals the presence of independent modifying gene effects influencing the type 1 diabetes risk encoded by the major HLA-DQB1, -DRB1 disease loci. Hum Mol Genet10:881-889

13. Buyse I, Sandkuyl LA, Zamani Ghabanbasani M et al. (1994) Association of particular HLA class II alleles, haplotypes and genotypes with susceptibility to IDDMin the Belgian population. Diabetologia 37:808-817

14. Baisch JM, Weeks T, Giles R, Hoover M, Stastny P, Capra JD (1990) Analysis of HLA-DQ genotypes and susceptibility in insulin-dependent diabetes mellitus. The $\mathrm{N}$ Engl J Med 322:1836-1841

15. Caillat-Zucman S, Djilali-Saiah I, Timsit J et al. (1997) Insulin dependent diabetes mellitus (IDDM): 12th International Histocompatibility Workshop study. In: Charron D (ed.) HLA. Proceedings of the Twelfth Histocompatibility Workshop and Conference, vol 2. EDK, Paris, pp 389398

16. Cucca F, Congia M, Angius E et al. (2001) A correlation between the relative predisposition of MHC class II alleles to type 1 diabetes and the structure of their proteins. Hum Mol Genet 10:2025-2037
17. Bell GI, Horita S, Karam JH (1984) A polymorphic locus near the human insulin gene is associated with insulindependent diabetes mellitus. Diabetes33:176-183

18. Chowdhury TA, Mijovic CH, Barnett AH (1999) The aetiology of Type I diabetes. Best Pract Res Clin Endocrinol Metab 13:181-195

19. Awata T, Kurihara S, Kikuchi C et al. (1997) Evidence for association between the class I subset of theinsulin gene minisatellite (IDDM2 locus) and IDDMin the Japanese population. Diabetes 46:1637-1642

20. Auwera B van der, Schuit F, Lyaruu I et al. (1995) Genetic susceptibility for insulin-dependent diabetes mellitus in Caucasians revisited: the importance of diabetes registries in disclosing interactions between HLA-DQ- andinsulin gene-linked risk. Belgian Diabetes Registry. J Clin Endocrinol Metab 80:2564-2565

21. Pugliese A, Zeller M, Fernandez A Jr et al. (1997) The insulin gene is transcribed in the human thymus and transcription levels correlated with allelic variation at the INS VNTR-IDDM2 susceptibility locus for type 1 diabetes. Nat Genet 15:293-297

22. Vafiadis P, Bennett ST, Todd JA et al. (1997) Insulin expression in human thymus is modulated byINS VNTR alleles at the IDDM2 locus. Nat Genet 15:289-292

23. Graham J, Hagopian WA, Kockum I et al. (2002) Genetic effects on age-dependent onset and islet cell autoantibody markers in Type 1 diabetes. Diabetes 51:1346-1355

24. Colman P, Steele C, Couper JJ et al. (2000) Islet autoimmunity in infants with a Type I diabetic relative is common but is frequently restricted to one autoantibody. Diabetologia 43:203-209 